\title{
Clinical and electrophysiological evaluation of nerve function impairment following cessation of multidrug therapy in leprosy
}

\author{
G. SAMANT, V. P. SHETTY, M. W. UPLEKAR \\ \& N. H. ANTIA \\ The Foundation for Medical Research, 84-A, \\ R.G. Thadani Marg, Worli, Bombay 400018 India
}

\section{Accepted for publication 21 October 1998}

\begin{abstract}
Summary Seventeen multibacillary (MB) and 15 paucibacillary (PB) cases of leprosy who had had regular and adequate multidrug therapy (MDT) were examined clinically and electrophysiologically at periodic intervals for 1 year following cessation of MDT. All the major nerves were assessed for nerve function impairment (NFI). Overall, two MB (13.3\%) and three PB (20\%) cases showed signs of deterioration clinically and/or electrophysiologically. The nerve conduction (NC) follow-up studies revealed no significant improvement in the sensory conduction in both the MB and PB groups of nerves, whilst motor conduction showed a significant improvement at the first 6-monthly follow-up among the $\mathrm{MB}$ group of nerves. At the study onset, sensory impairment $(\mathrm{MB}=62 \%, \mathrm{~PB}=25 \%)$ predominated over motor in terms of both severity and frequency. The lower extremity was more frequently and severely affected than the upper in both groups of patients. As an individual test, NC measurement proved to be more sensitive in detecting NFI, but the combination of physical palpatation for nerve thickening and graded nylon test (GNT) was closely comparable to measurement of nerve conduction.
\end{abstract}

\section{Introduction}

It is well recognized that care of individuals contracting leprosy has to extend beyond antibacterial treatment as adequate treatment with MDT does not necessarily ensure clearance of bacteria or bacterial antigens. ${ }^{1,2}$ Presence of viable bacteria and/or their antigens, particularly within the peripheral nerves, may lead to progressive involvement of peripheral nerves. ${ }^{3,4}$ Many issues related to this persistence of bacteria and their antigens have remained inadequately addressed. Does the residual nerve damage persist for long periods or is there a gradual natural recovery? Does it progress further and cause continued impairment of nerve function? Is the post-MDT nerve involvement in paucibacillary (PB) disease any different from that in multibacillary (MB) disease?

The present longitudinal study carried out among treated leprosy patients was designed to address the above questions. A group of $\mathrm{MB}$ and $\mathrm{PB}$ leprosy cases were examined

Correspondence to: V. P. Shetty 
clinically and subjected to electrophysiological studies at periodic intervals following completion of WHO-recommended multidrug therapy (MDT) with the objective of evaluating nerve function as well as the effectiveness of MDT in arresting nerve damage.

\section{Materials and methods}

Seventeen multibacillary cases (BL/LL) treated for a minimum of 2 years and 15 paucibacillary cases (TT/BT) treated for a minimum period of 6 months with the WHO-recommended MDT regimen, i.e. rifampicin (RFP) $600 \mathrm{mg}$ and $300 \mathrm{mg}$ clofazimine (CLF) once a month and CLF $50 \mathrm{mg}$ and diaminodiphenyl sulphone (DDS) $100 \mathrm{mg}$ daily for MB and RFP $600 \mathrm{mg}$ once a month and DDS $100 \mathrm{mg}$ daily for PB cases of leprosy ${ }^{5}$ were included in the study. Patient characteristics are given in Table 1.

\section{CLINICAL EXAMINATION}

All the patients were examined thoroughly by an experienced clinician to record number, size and activity of lesions and skin infiltration. All peripheral nerves were palpated to record thickness and tenderness if any. All major groups of muscles supplied by the median, ulnar, common perineal and common tibial were also tested and graded from 0 to 5 using the MRC, UK scale. ${ }^{6}$

\section{SENSORY TESTING}

Semi-quantitative testing for sensations was carried out using Semmes Weinstein graded nylon filaments, numbers $3 \cdot 2-6 \cdot 6$, on fixed points on hands and feet as described by Pearson. ${ }^{6}$ Use of graded nylon filaments has been shown to be simple, sensitive, reliable and reproducible. $^{7-9}$ The normal values for dorsal and palmer aspect of hand were $\leq 3.84$ and for the lateral and solar aspect of foot $=\leq 4.9$. Any values more than the above or, if doubtful,

Table 1. Study material at onset (1), first (2) and second (3) 6-month follow-up

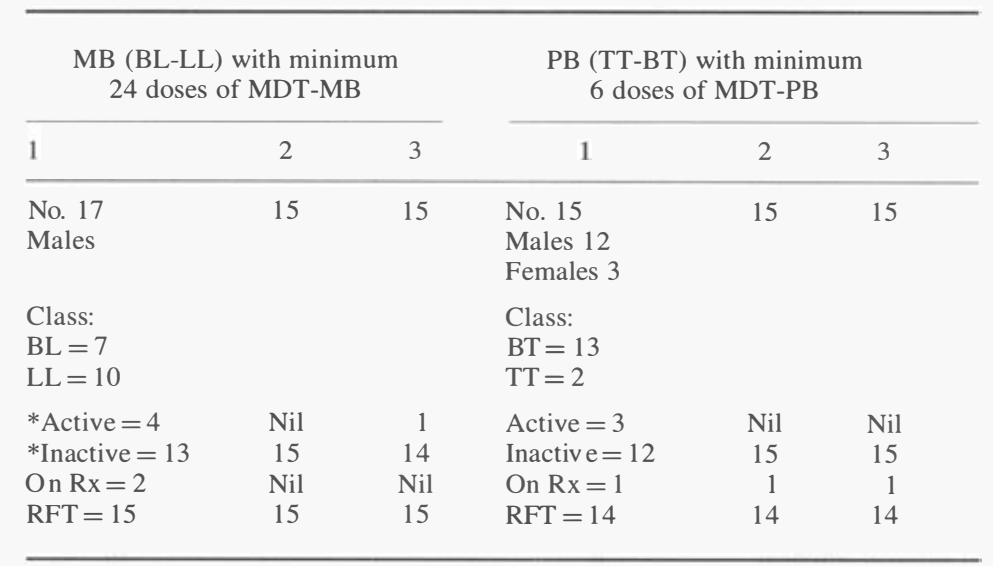

*Refers to skin lesional activity.

$\mathrm{RFT}=$ released from $\mathrm{Rx}$. 
an obvious difference to the contralateral side was considered abnormal. Nerve conduction studies help to evaluate the functional status of large and medium sized myelinated fibres, and a good correlation between electrophysiology and graded nylon test results is expected.

\section{BACTERIOLOGICAL EXAMINATION}

Slit skin smears were taken from six different sites to include at least one active lesion (when present), both ear lobes, and in addition any three other sites on the body. The smears were examined for the bacterial index (BI) and morphological index (MI). A lepromin test using Mitsuda lepromin was carried out on all the patients.

\section{ELECTROPHYSIOLOGICAL STUDIES}

Standard procedures were employed for recording sensory and motor action potentials for all peripheral nerves, using a Medelec MS92 EMG machine with an averager.

Sensory studies (SAP) were carried out on both left and right ulnar, median, radial, superficial peroneal and sural nerves. Sensory action potentials were recorded using surface electrodes. Motor studies (MAP) were carried out on ulnar, median, common peroneal and common tibial nerves.

Limits of normal values used for latency, amplitude and conduction velocity for sensory and motor nerves are shown in Table 2 (provided by Dr Shobha Pandya, EMG Department, Bombay Hospital). Values more than normal for latency and values less than normal limits for amplitude and conduction velocity (CV) recorded above were considered abnormal.

\section{BIOPSIES}

After the first clinical and electrophysiological recordings, a deep incision skin biopsy and a nerve biopsy were obtained from all the patients using local anaesthesia and with informed consent. Skin biopsies were taken from lesions that appeared most active, showing erythema and/or oedema. In the absence of active lesions, the choice of biopsy in the MB patients was

Table 2. Limit of normal values for sensory and motor nerves

\begin{tabular}{lccc}
\hline Sensory & $\begin{array}{c}<\text { Latency } \\
(\mathrm{ms})\end{array}$ & $\begin{array}{c}\text { Amplitude } \\
(\mathrm{mv})\end{array}$ & \\
\hline Ulnar & $3 \cdot 5$ & 3 & \\
Median & $3 \cdot 8$ & $7 \cdot 5$ & \\
Radial & $2 \cdot 8$ & 15 & \\
Sup. peroneal & 4 & 1 & Conduction \\
Sural & $3 \cdot 8$ & 5 & velocity \\
\hline & $<$ Latency & $>$ Amplitude & 40 \\
Motor & $(\mathrm{ms})$ & $(\mathrm{mv})$ & 39 \\
\hline Ulnar & 4 & 5 & 37 \\
Median & 4 & 5 & \\
Common peroneal & 6 & 1 & \\
Common tibial & 6 & 5 & \\
& & & \\
\hline
\end{tabular}


of the ear lobes. In PB cases with no active lesions, the biopsy was taken from an inactive lesion. Selection of peripheral nerve for biopsy was done on the basis of clinical and electrophysiological evidence of involvement. While one of the sural nerves was biopsied in all the MB cases, in PB cases the selection of the nerve to be biopsied varied according to the site of involvement and included sural (6) cutaneous branch of radial (7) cutaneous branch of ulnar (1) lateral thoracic (1) and superficial peroneal (1).

Paraffin-embedded sections stained with Trichrome modified fite ferraco (TRIFF) and anti-BCG using the sandwich immunoperoxidase assay were analysed for classification of leprosy, activity of disease and extent of antigen present.

\section{FOLLOW-UP STUDIES}

Clinical, bacteriological and electrophysiological assessments were repeated twice in each case at 6-month intervals, to record signs of clinical and functional improvement or deterioration, if any.

\section{Results}

CLINICAL FINDINGS AT ONSET

\section{Disease activity}

Four of $17 \mathrm{MB}$ cases and three of 15 PB cases had lesions which were clinically active, whereas all others were inactive at the time of recruitment.

\section{Lepromin reaction}

All the MB cases were lepromin negative, whereas all except one of the PB cases were lepromin positive.

NERVE THICKENING (SEE TABLE 11)

Sensory and motor impairment testing using graded nylon (GNT) and voluntary muscle testing (VMT) (see Tables 3, 4)

Among the MB cases, impaired sensory function was recorded in 90 of 170 nerves

Table 3. Results of graded nylon test in PB and MB cases at onset

\begin{tabular}{llllll}
\hline & \multicolumn{2}{c}{ PB cases $(n=15)$} & & \multicolumn{2}{c}{ MB cases $(n=17)$} \\
\cline { 2 - 3 } \cline { 5 - 6 } Nerve & Normal & Impaired & & Normal & Impaired \\
\hline Ulnar & $27(90 \%)$ & $3(10 \%)$ & & $16(47 \%)$ & $18(53 \%)$ \\
Median & $28(93 \%)$ & $2(7 \%)$ & & $17(50.0 \%)$ & $17(50.0 \%)$ \\
Radial & $26(87 \%)$ & $4(13 \%)$ & & $17(50.0 \%)$ & $17(50.0 \%)$ \\
$\begin{array}{l}\text { Sup. peroneal } \\
\text { Sural }\end{array}$ & $30(100 \%)$ & $0(0 \%)$ & & $17(50.0 \%)$ & $17(50.0 \%)$ \\
Total & $29(97 \%)$ & $1(3 \%)$ & & $13(38 \%)$ & $21(62 \%)$ \\
& $140(93 \%)$ & $10(7 \%)$ & & $80(47 \%)$ & $90(53 \%)$ \\
\hline
\end{tabular}


Table 4. Voluntary muscle function test in $17 \mathrm{MB}$ cases at onset

\begin{tabular}{lcc}
\hline Nerve & Normal & Impaired \\
\hline Ulnar & 33 & 1 \\
Median & $28(82 \%)$ & $6(18)$ \\
Common peroneal & 32 & 2 \\
Common tibial & 32 & 2 \\
Total & $125(92 \%)$ & $11(8 \%)$ \\
& & \\
\hline
\end{tabular}

Note: only one ulnar nerve among 15 PB cases showed impaired VM function.

examined. Sural nerve involvement was most frequently recorded (21/34), followed by the ulnar (18/34). The median, radial and superficial peroneal showed involvement of comparable frequency (17/34) each. Of the 136 nerves examined for motor function (VMT) only $11(8 \%)$ showed impairment, the median nerve being the most frequently involved (18\%).

Among the PB cases 10/150 nerves showed sensory impairment. Radial nerve was more frequently involved (4), followed by the ulnar (3) median (2) and sural (1) in this order. Motor impairment was noted only in one ulnar nerve.

\section{Electrophysiological findings on first examination (see Tables 5, 6): MB cases}

Of the 170 sensory recordings and 136 motor recordings carried out in $17 \mathrm{MB}$ patients, it was noted that the sensory involvement predominated over motor. In 77 (45\%) of the nerves there was no sensory action potential (SAP) and another $29(17 \%)$ showed abnormal sensory recordings (latency and amplitude). Thus, a total of 106 sensory nerves, i.e. $62 \%$ were impaired.

Among the sensory nerves, the sural nerve was the most frequently affected, followed by superficial peroneal, median, ulnar and radial in this order. However, the absence of sensory recordings was recorded most frequently in the superficial peroneal nerve. Whilst 54 (40\%) nerves showed abnormal motor recordings, total absence of motor function was not detected in any of the 136 nerves examined.

Among the motor nerves, the common peroneal was the most frequently affected.

Table 5. Sensory conduction results in PB and MB cases at onset

\begin{tabular}{|c|c|c|c|c|c|c|c|c|}
\hline \multirow[b]{2}{*}{ Nerve } & \multicolumn{4}{|c|}{ PB cases $(n=15)$} & \multicolumn{4}{|c|}{ MB cases $(n=17)$} \\
\hline & Normal & Impaired & Absent & Total impaired & Normal & Impaired & Absent & Total impaired \\
\hline Ulnar & 23 & 4 & 3 & 7 & 16 & 4 & 14 & 18 \\
\hline Median & 22 & 6 & 2 & 8 & 13 & 12 & 9 & 21 \\
\hline Radial & 22 & 5 & 3 & 8 & 16 & 9 & 9 & 18 \\
\hline Sup. peroneal & 23 & 0 & 7 & 7 & 10 & 0 & 24 & 24 \\
\hline Sural & 23 & 5 & 3 & 8 & 9 & 4 & 21 & 25 \\
\hline Total & $\begin{array}{l}113 \\
(75 \cdot 3 \%)\end{array}$ & $\begin{array}{l}20 \\
(13 \cdot 3 \%)\end{array}$ & $\begin{array}{l}17 \\
(11 \cdot 3 \%)\end{array}$ & $\begin{array}{l}38 \\
(25 \%)\end{array}$ & $\begin{array}{l}64 \\
(38 \%)\end{array}$ & $\begin{array}{c}29 \\
(17 \%)\end{array}$ & $\begin{array}{l}77 \\
(45 \%)\end{array}$ & $\begin{array}{l}106 \\
(62 \%)\end{array}$ \\
\hline
\end{tabular}


Table 6. Motor conduction results in $\mathrm{PB}$ and $\mathrm{MB}$ cases at onset

\begin{tabular}{|c|c|c|c|c|c|c|c|c|}
\hline \multirow[b]{2}{*}{ Nerve } & \multicolumn{4}{|c|}{ PB cases $(n=15)$} & \multicolumn{4}{|c|}{ MB cases $(n=17)$} \\
\hline & Normal & Impaired & Absent & Total impaired & Normal & Impaired & Absent & Total impaired \\
\hline Ulnar & 26 & 3 & 1 & 4 & 23 & 11 & 0 & 11 \\
\hline Median & 26 & 4 & 0 & 4 & 24 & 10 & 0 & 10 \\
\hline Common peroneal & 25 & 5 & 0 & 5 & 12 & 22 & 0 & 22 \\
\hline Common tibial & 27 & 3 & 0 & 3 & 23 & 11 & 0 & 11 \\
\hline Total & $\begin{array}{l}104 \\
(87 \%)\end{array}$ & $\begin{array}{l}15 \\
(12 \cdot 4 \%)\end{array}$ & 1 & $\begin{array}{l}16 \\
(13 \%)\end{array}$ & $\begin{array}{l}82 \\
(60 \%)\end{array}$ & $\begin{array}{l}54 \\
(40 \%)\end{array}$ & 0 & $\begin{array}{l}54 \\
(40 \%)\end{array}$ \\
\hline
\end{tabular}

Common tibial, ulnar and median showed involvement of comparable frequency. The lower extremity was more frequently and severely affected than the upper, for both sensory and motor functions.

\section{$P B$ cases}

Of the 150 nerves tested for SAP from 15 patients, no SAP was recorded in 17 . Twenty were abnormal (delayed), while the rest were within the normal limits $(75 \%)$.

Of the 121 motor recordings, only one nerve showed absent motor action potential. Fifteen were abnormal and the rest were normal. As in the MB group, sensory involvement predominated over motor and the lower extremity was more commonly involved as compared to the upper. The radial, ulnar, median, sural and superficial peroneal nerves showed involvement of comparable frequency. Among the sensory nerves in the PB group also, the superficial peroneal nerve was the most severely affected (7/30 absent). Among the motor nerves, the lateral popliteal was the most commonly affected (5/31) while the median, ulnar and posterior tibial nerves showed involvement of comparable frequency.

\section{FOLLOW-UP RESULTS}

All investigations except biopsies were repeated twice at 6-month interval. Only those patients, with complete follow-up data were considered for the final analysis.

\section{CLINICAL FINDINGS}

$M B$ cases

Fifteen MB cases were available for the final analysis, of whom 11 had unchanged GNT and VMT, two showed marginal sensory improvement and two showed sensory/motor deterioration.

Of the four MB cases whose patches were recorded as clinically active at onset, three became clinically inactive at the second 6-month follow-up, i.e. after 1 year. The fourth patient was recorded as inactive at the first 6-month follow-up, but showed active skin infiltration along with deterioration in GNT charting at the second 6-month follow-up. 
$P B$ cases

Of the 15 PB cases, nine showed no change, three improved and three deteriorated neurologically.

The deterioration among the three PB cases was in the form of development of an active skin lesion, recurrent ulnar and median neuritis with appearance of a new skin lesion and recurrent abscess formation in one of the ulnar nerves. The patient with a new skin lesion was lost for follow-up, while the one with recurrent neuritis continued to show progressive deterioration despite a full course of MDT-MB, after having taken a course of MDT-PB. The patient with ulnar nerve abscess had absent sensory conduction in the nerve from the first NC examination.

Three of $15 \mathrm{BT}$ cases where patches were recorded as active at onset became inactive at the second follow-up, one of them having received 9 months of MDT-PB.

\section{Bacteriological follow-up findings}

In all except two patients, there was a significant decline in the smear BI during the follow-up investigations. In two cases the smear BI remained static around $3+$.

All the PB cases were smear negative at onset and they remained negative during the follow-up.

\section{Electrophysiological follow-up (sensory and motor) findings (see Tables 7, 8, 9 and 10)}

In order to determine whether there was any significant overall improvement or deterioration in either sensory or motor conduction recordings during the two 6-month follow-ups, the total numbers of normal recordings at three intervals were compared. The biopsied nerves were

Table 7. Sensory conduction follow-up - MB

\begin{tabular}{lcccc}
\hline & Normal & Impaired & Absent & Total impaired \\
\hline Initial & $54(40 \%)$ & $29(22 \%)$ & $52(39 \%)$ & $81(60 \%)$ \\
Second & $60(44 \%)$ & $23(17 \%)$ & $52(39 \%)$ & $75(56 \%)$ \\
Final & $62(46 \%)$ & $24(17 \%)$ & $49(36 \%)$ & $73(54 \%)$ \\
\hline
\end{tabular}

No significant differences were observed.

Table 8. Motor conduction follow-up - MB

\begin{tabular}{lrrcr}
\hline & Normal & Impaired & Absent & Total impaired \\
\hline Initial & $76(63 \%)$ & $43(36 \%)$ & 1 & $44(37 \%)$ \\
Second & $102(85 \%)$ & $12(14 \%)$ & 1 & $13(15 \%)$ \\
Final & $105(88 \%)$ & $14(12 \%)$ & 1 & $15(12 \%)$ \\
\hline
\end{tabular}

A highly significant critical ratio $=3.9611(p=0.01)$ was observed between the initial and second visits. 
Table 9. Sensory conduction follow-up - PB

\begin{tabular}{lrlrr}
\hline & Normal & Impaired & Absent & Total impaired \\
\hline Initial & $109(79 \%)$ & $18(13 \%)$ & $11(8 \%)$ & $29(21 \%)$ \\
Second & $98(83 \%)$ & $10(8 \%)$ & $10(8 \%)$ & $20(17 \%)$ \\
Final & $116(84 \%)$ & $11(8 \%)$ & $11(8 \%)$ & $22(16 \%)$ \\
\hline
\end{tabular}

No significant differences were observed.

Table 10. Motor conduction follow-up - PB

\begin{tabular}{lcccc}
\hline & Normal & Impaired & Absent & Total impaired \\
\hline Initial & $91 \%$ & $8 \%$ & $1 \%$ & $9 \%$ \\
Second & $94 \%$ & $3 \%$ & $3 \%$ & $6 \%$ \\
Final & $93 \%$ & $4 \%$ & $3 \%$ & $7 \%$ \\
\hline
\end{tabular}

No significant differences were observed.

excluded in this analysis. The total number of normal sensory recordings at the onset, first and second follow-up or between onset and second follow-up showed no statistically significant differences in both the MB and PB group of nerves. It was therefore concluded that there was no statistically significant improvement in the sensory conduction velocity in both MB and PB patients studied. However, the motor recordings showed a significant improvement at the first 6-month follow-up [critical ratio $(\mathrm{CR})=3.9611, p=0.01$ ] and remained steady at the second 6-month follow-up among the MB group of nerves. No significant improvement was noted in the motor recordings among the PB nerves.

\section{Clinicoelectrophysiological correlation at onset (see Table 11)}

Among the MB group of patients, of the 238 nerves tested at the study onset $82(34.5 \%)$ were recorded as thickened on physical palpation, 94 (39.5\%) nerves were recorded as abnormal using graded nylon whereas 139 (58.4\%) nerves were recorded as abnormal using electrophysiology. Among the PB group of patients, of the 210 nerves tested 27 (12.9\%) were palpably thick, $10(4.8 \%)$ showed graded nylon abnormality and $45(21.4 \%)$ of the nerves were abnormal electrophysiologically. Thus electrophysiological recordings revealed a significantly higher number of involved nerves as compared to either physical palpation or the graded nylon test in MB as well as the PB group.

\section{Discussion}

In the present study, a group of 15 each of multibacillary (BL/LL) and paucibacillary (TT/BT) cases of leprosy were assessed for change in nerve functions for a period of 1 year following cessation of MDT. Two MB (13.3\%) and three PB (20\%) cases showed signs of deterioration in nerve function clinically and/or electrophysiologically. 
Table 11. Clinicoelectrophysiological correlation at onset

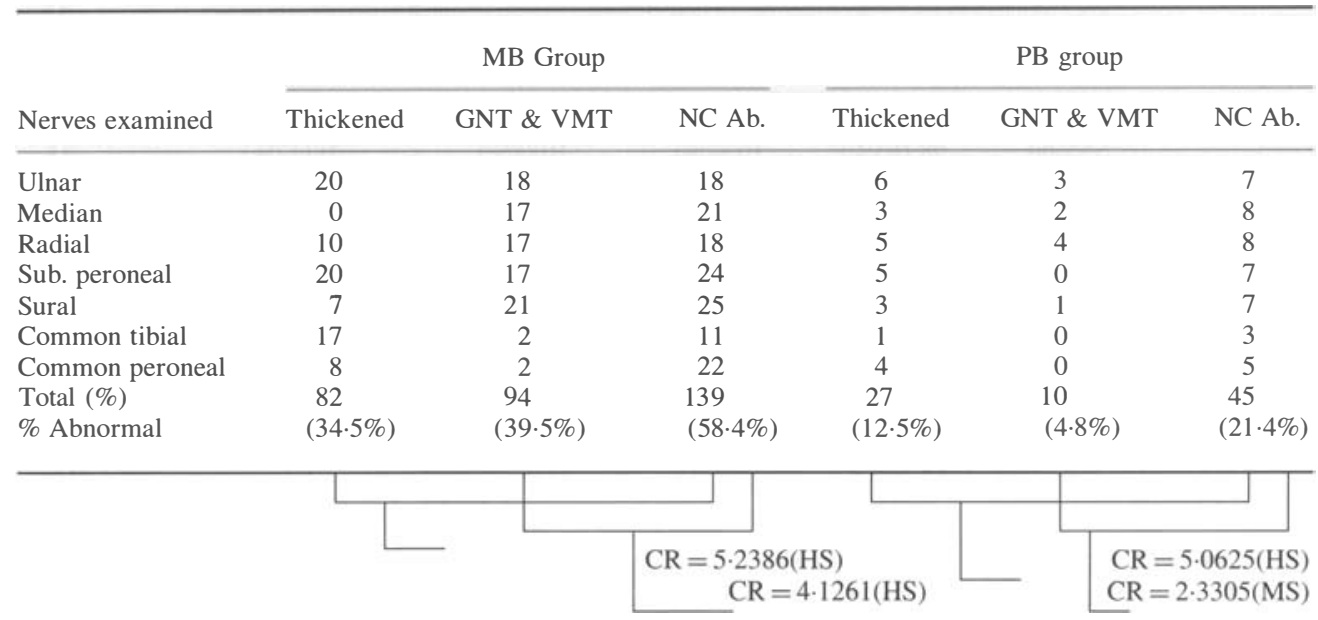

Ab. = abnormal; GNT = graded nylon test $\mathrm{NC}=$ nerve conduction study; $\mathrm{VMT}=$ voluntary muscle testing; $\mathrm{CR}=$ critical ratio; HS = highly significant: $(p<0 \cdot 01) ; \mathrm{MS}=$ marginally significant: $(p=0 \cdot 05)$.

The electrophysiological follow-up studies in MB and PB cases of leprosy during the first year of cessation of MDT showed no significant improvement in the sensory conduction in both $\mathrm{MB}$ and $\mathrm{PB}$ group of nerves. However, the motor conduction showed significant improvement, though only among the MB group of patients at the first 6-month follow-up. It remained static at the second follow-up. This was not surprising, as the motor conduction was least affected at the onset and unlike PB nerves, there were a large number of nerves $(40 \%)$ that had shown mild involvement. The reversal of delay in motor conduction in these nerves could be attributed to subsidence of inflammation and oedema and/or reduction in bacterial antigens and immune complexes.

The bacterial load declines in the skin at a steady rate following cessation of MDT. ${ }^{5} \mathrm{~A}$ similar trend was noted in the present study. However, such longitudinal data are not available for the peripheral nerves. Random studies carried out on nerves show prolonged persistence of bacteria and antigens even in patients who were consistently smear negative. ${ }^{1-3}$ Thus subsidence of oedema and inflammation is the most likely mechanism for the improvements found in motor conduction velocity.

As mentioned in the methodology, nerve biopsies obtained at the onset from this group of patients were studied using both light and electron microscope. The results are documented elsewhere. ${ }^{10}$ The regenerating/remyelinating myelinated fibres were seen in large numbers in several of the nerves studied. However, this is not a hallmark feature of treated nerves, as regenerating/remyelinating fibres were often seen in actively involved nerves of untreated cases of leprosy. ${ }^{11} \mathrm{~A}$ majority of the nerves that were biopsied were grossly involved sural nerves showing no recordable sensory action potential. Also, a majority of the nerves showing absent sensory action potential at the onset remained so throughout the follow-up. It can be extrapolated, therefore, that the regenerating/remyelinating activity noted in the biopsied nerves was not functionally reflected in the $\mathrm{NC}$ and/or clinical recordings. One of the explanations could be that the remyelination was incomplete or discontinuous, or there was collateral sprouting. In both situations, an inordinate delay in the propagation of action 
potentials would result in absent sensory action potentials. None of the tests used in the present study was sensitive enough to gauge the extent of remyelination or further worsening in these nerves.

Special mention must be made of the two MB cases who had shown deterioration in nerve function during the follow-up. One MB case showed simultaneous sensory deterioration and a new active skin lesion at the second follow-up. The patient was restarted on MDT-MB for 1 year; the response was good and the skin lesion subsided. The second MB patient, who deteriorated to develop glove and stocking type of anaesthesia, remained so at the subsequent clinical follow-up.

As expected, the sensory involvement predominated over motor, both in terms of severity and frequency. The lower extremity was more severely and frequently involved than the upper in both MB and PB cases of leprosy. Among the sensory nerves in the MB group, the sural nerve was found to be the most frequently involved $(73.5 \%)$, followed by the superficial peroneal nerve (70.5\%). The latter was also found to be the most severely affected nerve (all absent recordings). The median (62\%), ulnar (53\%) and radial nerves (53\%) were next in order. Among the motor nerves, the common peroneal was the most frequently affected nerve $(65 \%)$. The common tibial, ulnar and the median nerves showed involvement of comparable frequency $(50 \%$ each).

There was a good concordance between clinical and electrophysiological abnormalities noted in the nerves examined, in that all the clinically involved nerves $(80 / 150=47 \%)$ showed electrophysiological involvement. Besides, 22/150 (14\%) sensory nerves and 15/120 $(12.5 \%)$ motor nerves that were clinically normal showed $\mathrm{NC}$ abnormality, proving NC to be more sensitive in detecting sensory and motor impairment. Biopsy of few such nerves obtained in this study confirmed histologically demonstrable lesions in these nerves.

Significantly in the present study, a combination of physical palpation and use of graded nylon could collectively detect involvement of $32.8 \%$ of nerves, only marginally less than that detected by nerve conduction studies $(41 \cdot 1 \%)$. It demonstrates that under field conditions, combination of the above two tests could serve as the most useful tool for detecting the nerve function impairment. Electrophysiology should be recommended only under special circumstances for further confirmation or as a research tool.

\section{Acknowledgements}

We are grateful to Dr R. Ganpati, Director, Bombay Leprosy Project for referring some of the patients for this study, for the unfailing co-operation from the patients and from staff of FMR neurology group. Fruitful guidance, normal values and generous use of an EMG machine were provided by Dr Shobha Pandya of Bombay Hospital. Dr Satish Arolkar did all the biopsies. Typing was done by Mrs Jayalakshmy and Ms Anagha carried out the statistical analysis. We are indebted to Lepra, UK for financial support.

\section{References}

${ }^{1}$ Shetty VP, Suchitra K, Uplekar MW, Antia NH. Higher incidence of viable Mycobacterium leprae within the nerve as compared to skin among multibacillary leprosy patients released from multidrug therapy. Lepr Rev, 1997; 68: $131-138$. 
2 Shetty VP, Suchitra K, Uplekar MW, Antia NH. Persistence of Mycobacterium leprae in the peripheral nerve as compared to skin of multidrug treated leprosy patients. Lepr Rev, 1992; 63: 329-336.

3 Shetty VP, Uplekar MW, Antia NH. Immunohistological localization of mycobacterial antigens within the peripheral nerves of treated leprosy patients and their significance to nerve damage in leprosy. Acta Neuropathol, 1994; 88: 300-306.

4 Van Brakel WH, Khawas IB. Silent neuropathy in leprosy: an epidemiological description. Lepr Rev, 1994; 65: $350-360$.

5 WHO. Study group on chemotherapy of leprosy for control programmes. World Health Organisation, 1982: TRS 675.

6 Pearson JMH. The evaluation of nerve damage in leprosy. Lepr Rev, 1982; 53: 119-130.

7 Antia NH, Mehta LN, Shetty VP, Irani PF. Clinical, electrophysiological, quantitative, histologic and ultrastructural studies of the index branch of the radial cutaneous nerve in leprosy. I. Preliminary report. Int J Lepr, 1975; 43: 106-113.

8 Mehta LN, Shetty VP, Antia NH, Irani PF. Quantitative, histologic and ultrastructural studies of the index branch of the radial cutaneous nerve in leprosy and its correlation with electrophysiologic study. Int J Lepr, 1975; 43: $256-264$.

9 Bell-Krotoski JA. 'Pocket' filaments and specifications for the Semmes-Weinstein monofilaments. J Hand Ther, 1990; Jan-Mar: 26-31.

10 Jacobs JM, Shetty VP, Antia NH. A morphological study of nerve biopsies from cases of multibacillary and paucibacillary leprosy given multidrug therapy. Acta Neuropathol, 1993; 85: 533-541.

11 Shetty VP, Antia NH, Jacobs JM. The pathology of early leprous neuropathy. J Neurol Sci, 1988; 88: 115-131. 